\title{
GOLGA2/GM130 is a Novel Target for Neuroprotection Therapy in Intracerebral Hemorrhage
}

\section{Shuwen Deng}

Second Xiangya Hospital

Qing Hu

Second Xiangya Hospital

Qiang He

Second Xiangya Hospital

\section{Xiqian Chen}

Second Xiangya Hospital

Wei Lu ( $\square$ luwei0338@csu.edu.cn )

Second Xiangya Hospital, central south university https://orcid.org/0000-0002-3760-1550

\section{Research Article}

Keywords: Golgi apparatus, therapy, intracerebral hemorrhage, autophagy, blood-brain barrier

Posted Date: June 1st, 2021

DOI: https://doi.org/10.21203/rs.3.rs-547422/v1

License: (c) (i) This work is licensed under a Creative Commons Attribution 4.0 International License. Read Full License 


\section{Abstract}

Blood-brain barrier (BBB) impairment after intracerebral hemorrhage (ICH) can lead to secondary brain injury and aggravate neurological deficits. Currently, there are no effective methods for its prevention or treatment partly because of to our lack of understanding of the mechanism of ICH injury to the BBB. Here, we explored the role of Golgi apparatus protein GM130 in the BBB and neurological function after ICH. The levels of the tight junction-associated proteins ZO-1 and occludin decreased, whereas those of LC3-II, an autophagosome marker, increased in hemin-treated Bend. 3 cells $(p<0.05)$. Additionally, GM130 overexpression increased ZO-1 and occludin levels, while decreasing LC3-II levels $(p<0.05)$. GM130 silencing reversed these effects and mimicked the effect of hemin treatment $(p<0.05)$. Moreover, tight junctions were disrupted after hemin treatment or GM130 silencing and repaired by GM130 overexpression. GM130 silencing in Bend. 3 cells increased autophagic flux, whereas GM130 overexpression downregulated this activity. Furthermore, GM130 silencing-induced tight junction disruption was partially restored by 3-methyladenine (an autophagy inhibitor) administration. Similarly, an in vivo $\mathrm{ICH}$ rat model showed elevated perihematomal ZO-1 and occludin expression and decreased LC3-II expression $(p<0.05)$; these results were reversed following GM130 silencing $(p<0.05)$. Perihematomal Evans Blue staining and brain water content were elevated in GM130-silenced ICH rats relative to control ICH rats. GM130 overexpression can protect BBB integrity from brain injury, inhibit excessive autophagy flux in $\mathrm{ICH}$, and improve neurobehavioral prognosis. Therefore, therapy targeting GM130 regulation might represent a potential treatment for acute brain injury after $\mathrm{ICH}$.

\section{Introduction}

Spontaneous intracerebral hemorrhage $(\mathrm{ICH})$ is a potentially fatal cerebrovascular disease accompanied by a high mortality rate. Brain injury after $\mathrm{ICH}$ can be divided into primary and secondary brain injury [1]. Blood components and blood-cell degradation products from ruptured blood vessels can cause severe secondary brain injury [2], and blood-brain barrier (BBB) impairment after ICH is an important pathophysiological mechanism [3]. BBB damage can lead to secondary brain injury and aggravate neurological deficits $[4,5]$. Over the recent decades, no new effective methods have emerged to prevent $\mathrm{ICH}$ from injuring the BBB [6]; therefore, a comprehensive understanding of the mechanisms underlying BBB injury is needed to guide the development of new therapeutic methods.

The BBB functions as a physical and physiological barrier between the blood and brain, and disruption of BBB integrity is a well-documented cause of brain edema following a variety of brain injuries [7]. BBB integrity is damaged under oxidative stress and inflammatory conditions. Additionally, an increase in autophagic flux after stroke can affect BBB integrity [8]; however, whether its effect is destructive or protective remains controversial $[9,10]$, with most studies suggesting that excessive autophagy can cause destruction of the BBB after ICH $[11,12]$.

Autophagy is generally enhanced under conditions of starvation, oxidative damage [13], endoplasmic reticulum stress [14], or Golgi stress [15]. Defects in autophagic flux can lead to accumulation of 
damaged or aging proteins and thereby play a critical role in the pathogenesis of several diseases, including neurodegenerative diseases [16], cardiovascular diseases [17], metabolic diseases [18], and cancer [19]. Excessive autophagosome formation is induced by stress conditions, such as oxidative stress, energy and nutrient deprivation, and pro-apoptotic conditions, and can also be regulated by Golgi structure proteins, such as GM130 and Grasp55.

GM130 is a cis-Golgi protein that plays a role in maintaining Golgi morphology and controlling protein glycosylation and vesicle transport. Inhibition of glycosylation caused by GM130 silencing can induce autophagy [20]. Further studies have revealed that GM130 regulates the autophagy signaling pathway by interacting with GABARAP [21,22]. Therefore, GM130 plays a role in maintaining the proper levels of autophagy. We previously observed that decreased GM130 levels lead to Golgi fragmentation in an in vivo model of $\mathrm{ICH}$ [23]; however, the specific role of $\mathrm{GM} 130$ in ICH pathogenesis remains unknown. Based on these findings, we hypothesized that targeted regulation of GM130 expression might rescue BBB integrity, which is mediated by influencing autophagic flux, thereby reducing secondary brain injury and neurological deficits after $\mathrm{ICH}$. Our findings will potentially provide a new treatment direction for BBB protection after $\mathrm{ICH}$.

\section{Materials And Methods Experimental design}

The experimental design is illustrated in Figure 1. In the first experiment, Bend. 3 cells were sorted into six groups and treated with various concentrations of hemin $(12.5,25,50,100$, and $200 \mu \mathrm{M})$ to assess the expression of GM130, LC3b, p62, occludin, and ZO-1 after ICH in vitro. Subsequently, Bend.3 cells were transfected with pcDNA3.1-GM130 or small-interfering (si)RNAs targeting GOLGA2/GM130 (siGOLGA2/siGM130) to determine the effect of GM130 silencing or overexpression on p62, LC3b, occludin, and ZO-1 expression under normal or ICH conditions. Additionally, Bend. 3 cells were treated with the autophagy inhibitor 3-methyladenine (3-MA) after transfection with siGOLGA2/siGM130 to investigate changes in occludin and ZO-1 expression.

In the second experiment, rats were used to quantify the effects of collagenase on early brain injury after $\mathrm{ICH}$. The rats were randomly and evenly assigned to five groups ( $\mathrm{n}=6$ rats/group): a sham group and ICH groups at various time points $(12,24,48$, and $72 \mathrm{~h})$.

In the third experiment, rats were used to evaluate the effects of siGM130 or adeno-associated virus (AAV)-mediated GM130 overexpression on early brain injury at $48 \mathrm{~h}$ after $\mathrm{ICH}$. For the first part, rats were randomly divided into five groups: sham, $\mathrm{ICH}, \mathrm{ICH}+$ vehicle-1 (transfection reagent), ICH+vehicle-2 [mixtures of transfection reagent and negative control (NC) siRNA diluent], and ICH+siGM130. For the second part, rats were randomly divided into four groups: sham, ICH, ICH+AAV-NC, and ICH+AAV-GM130. Garcia score $(n=6)$, brain water content $(n=6)$, Evans Blue (EB) extraction $(n=3)$, immunoblot $(n=6)$, and immunofluorescence (IF) staining $(n=3)$ analyses were performed for each rat. In the third part, to 
investigate the effects of autophagy inhibition by 3-MA on siGM130-induced tight junction damage, tissues from ICH rats pretreated with 3-MA in vivo were evaluated to observe the expression of tight junction protein via immunoblot analyses.

\section{Cells and cell culture}

Mouse brain endothelial cells (Bend.3) were purchased from Shanghai Zhong Qiao Xin Zhou Biotechnology company (ZQ-0090; Shanghai, China) [24]. Cells were cultured in Dulbecco's modified Eagle medium (DMEM; cat\#12900017; Life Technologies, Carlsbad, CA, USA) with 10\% fetal bovine serum (GIBCO, Gaithersburg, MD, USA) and 1\% penicillin-streptomycin (100 mg/mL penicillin and 100 $\mathrm{mg} / \mathrm{mL}$ streptomycin) at $37^{\circ} \mathrm{C}$ and $5 \% \mathrm{CO}_{2}$ [25]. Hemin (51280; Sigma-Aldrich, St. Louis, MO, USA) was dissolved in sodium hydroxide and then diluted with DMEM and administered to Bend. 3 cells to establish the in vitro $\mathrm{ICH}$ model.

\section{Animal model}

The study was conducted according to the guidelines for the care and use of laboratory animals of Central South University, and the experiment protocols were approved by the Central South University Animal Care and Use Committee (approval number: 2021013). Sprague-Dawley rats were housed at 25 ${ }^{\circ} \mathrm{C}$ with ad libitum access to water and food. The light in the room was controlled at a $12 \mathrm{~h} \mathrm{light/dark}$ cycle [5], and rats were randomly assigned to each group.

Rats were anesthetized with pentobarbital sodium $(45 \mathrm{mg} / \mathrm{kg})$ via intraperitoneal injection and then placed in a stereotaxic apparatus. Collagenase type IV $(0.2 \mathrm{U}$ in $1 \mu \mathrm{L}$ of sterile normal saline) was injected into the right basal ganglia of the rat brain with the following stereotaxic coordinates: $A P, 0.1 \mathrm{~mm} ; \mathrm{ML}, 3$ $\mathrm{mm}$; and DV, $6.0 \mathrm{~mm}$ to the bregma [26]. The sham-operated rats were administered sterile normal saline. Rats were then allowed to recover in separate cages with ad libitum access to food and water.

\section{RNAi-mediated GM130 silencing in vitro}

Before induction of ICH in vitro, cells were transfected with siGM130 duplex 01 (siGM130-1; GenePharma, Shanghai, China), duplex 02 (siGM130-2; GenePharma), or NC siRNA (siRNA-NC) with PepMute (SignaGen Frederick, MD, USA). Cells in the vehicle group received an equal volume of diethylpyrocarbonate-treated water. For western blot (WB) or IF analyses, cells were harvested $72 \mathrm{~h}$ after transfection.

\section{Plasmid construction and transfection in vitro}


The GM130 gene was extracted from Bend. 3 cells and cloned into the pcDNA3.1(+) vector. The GM130specific primers were as follows: 5'-ccaagctggctagc gctagcATGTGGCCCCCCCGCTTCCC-3' and 5'gtttaaacggge cctctagaTTATACAACCATGATCTTCA-3' [24]. Transfection was performed using Lipofectamine 3000 (cat\#L3000150; Invitrogen, Carlsbad, CA, USA), according to manufacturer instructions. At $12 \mathrm{~h}$ after transfection, Bend. 3 cells were treated with hemin for $36 \mathrm{~h}$. Cells were harvested for WB or IF analysis $72 \mathrm{~h}$ after transfection.

\section{siRNA transfection and AAV injection in vivo}

siGM130 or siRNA-NC (20 $\mu \mathrm{M}$; GenePharma) was mixed with EntransterTM-in vivo (Engreen, Beijing, China). Prior to in vivo ICH induction, the mixture (final volume: $5 \mu \mathrm{L}$ ) was injected into the right lateral ventricle (stereotaxic coordinates: $A P, 0.8 \mathrm{~mm}$; and $\mathrm{ML}, 1.5 \mathrm{~mm}$ right to the bregma and $4.5 \mathrm{~mm}$ ventral to the skull) at a flow rate of $0.5 \mu \mathrm{L} / \mathrm{min}$ through another $1 \mathrm{~mm}$ burr hole [26]. The rats in the NC-1 group received an intracerebroventricular injection of an equal volume of the transfection reagent before $\mathrm{ICH}$ establishment. The sham-operated rats were administered $5 \mu \mathrm{L}$ of sterile normal saline into the same position [1]. Next, 3-MA was dissolved in normal saline by heating the solution to $60^{\circ} \mathrm{C}-70^{\circ} \mathrm{C}$ immediately before treatment, followed by storage at $-20^{\circ} \mathrm{C}$. Rats received intracerebroventricular injections of 3-MA in the ipsilateral ventricle $(400 \mathrm{nmol} / \mu \mathrm{L})$ as a pretreatment $15 \mathrm{~min}$ before $\mathrm{ICH}$. Sham rats and siRNA-pretreated rats received an equivalent volume of vehicle. ICH models were established 3 days after siRNA transfection.

On day 20 before ICH establishment, $2.5 \mu$ L Flag-AAV-GM130 and Flag-AAV-NC $\left(3 \times 10^{11}\right.$ viral genomes $(\mathrm{vg}) / \mathrm{mL}$; GenePharma) were administered via intracerebroventricular injection. Sham-operated rats were administered $2.5 \mu \mathrm{L}$ of sterile normal saline into the same position.

\section{Evaluation of BBB permeability}

EB extravasation was performed as reported previously [27]. EB solution (2\%) in normal saline $(4 \mathrm{~mL} / \mathrm{kg})$ was administered into the femoral vein $48 \mathrm{~h}$ after ICH establishment. The dye was allowed to circulate for $2 \mathrm{~h}$, and then $200 \mathrm{~mL}$ of ice-cold phosphate-buffered saline (PBS) was used for trans-cardiac perfusion under deep anesthesia and collection in the right hemisphere of the brain. The supernatant of hemorrhaged brain in the right hemisphere was then mixed with an equal amount of trichloroacetic acid solution and incubated at room temperature for $1 \mathrm{~h}$, after which the samples were centrifuged for $30 \mathrm{~min}$ at $15,000 \mathrm{rpm}$ and $4{ }^{\circ} \mathrm{C}$ to separate the supernatant for quantification [28]. EB staining results were measured using a spectrophotometer (Spectronic Genesys 10 UV; Thermo Fisher Scientific, Waltham, MA, USA) at $610 \mathrm{~nm}$ and quantified with a standard curve [28].

\section{Garcia scores and measurement of brain water content}


We used Garcia's 18-point scale to assess neurological defects $48 \mathrm{~h}$ after $\mathrm{ICH}$. This was performed by two trained investigators in a blind controlled method, with examination of neurological status undertaken according to the standards listed in Table 1 [29]. Measurement of brain water content was performed as previously described [28]. Calculation of brain water content was as follows:

\section{Content $(\%)=[($ wet weight - dry weight $) /$ wet weight $] \times 100$}

\section{MTT assay and transmission electron microscopy (TEM) analysis}

An MTT assay was used to determine cell viability (Sigma-Aldrich) according to absorbance at $450 \mathrm{~nm}$ detected using a microplate reader (Bio-Rad Laboratories, Hercules, CA, USA) [30]. Briefly, Bend.3 cells were fixed in $2.5 \%$ glutaraldehyde at $4{ }^{\circ} \mathrm{C}$ for $24 \mathrm{~h}$. After treatment with $1 \% \mathrm{OsO}_{4}$ for $1 \mathrm{~h}$, Bend. 3 cells were washed with PBS for 30 min, dehydrated, and then embedded in epon-812 [31]. Ultrathin sections (40-70 $\mathrm{nm}$ ) were cut and mounted on pioloform-coated copper grids, followed by staining with lead citrate and uranyl acetate and observation through TEM (HT7700; Hitachi, Tokyo, Japan).

\section{IF and WB analyses}

IF staining was performed using anti-ZO-1 (Cat \#40-2200; Invitrogen), anti-occludin (Cat \#33-1500; Invitrogen), anti-GM130 (Cat \#610822; BD Bioscience, Franklin Lakes, NJ, USA), and anti-LC3b (Cat \#ab48394; Abcam, Cambridge, UK), as previously described [32]. For immunoblot analysis, proteins were separated on $8 \%$ to $10 \%$ gels using sodium dodecyl sulfate-polyacrylamide gel electrophoresis and transferred to polyvinylidene difluoride membranes, which were then blocked with Tris-buffered saline containing $5 \%$ skim milk for $1 \mathrm{~h} 25^{\circ} \mathrm{C}$. WB was performed after overnight incubation at $4{ }^{\circ} \mathrm{C}$ with the corresponding primary anti-ZO-1, anti-occludin, anti-GM130, anti-LC3b, and anti-p62 (ab109012; Abcam) antibodies.

\section{Statistical analysis}

Data are presented as the mean \pm standard deviation (SD). Statistical analyses were performed using GraphPad Prism software (v.8.0; GraphPad Software, San Diego, CA, USA) and SPSS (v.19.0; SPSS, Inc., Chicago, IL, USA). Data were evaluated using one-way analysis of variance to compare differences between multiple groups. Variance homogeneity was assessed using the least significant difference test, and absence of variance was assessed using Dunnett's test. A $p<0.05$ was considered significant.

\section{Results}




\section{Hemin downregulates GM130 expression, resulting in Golgi dysfunction, decreased tightjunction integrity, and attenuated autophagy induction in mouse brain endothelial cells}

We assessed GM130 expression and Golgi morphology after hemin treatment for $48 \mathrm{~h}$. As shown in Figure2a and 2c, GM130 levels decreased with increasing concentrations of hemin, with a significant decrease in GM130 levels observed at $100 \mu \mathrm{M}$ hemin $(p<0.05)$. IF revealed dispersal of GM130-marked Golgi apparatus in the cytoplasm, with some undergoing fragmentation after hemin treatment (Fig. 2b). Additionally, $100 \mu \mathrm{M}$ hemin significantly decreased expression levels of ZO-1 $(p<0.05)$ and occludin $(p<$ 0.05; Fig. 2a and c). IF staining further revealed discontinuity in tight junctions (Fig. 2b). Moreover, we observed that LC3-II expression levels increased $(p<0.05)$ and p62 decreased $(p=0.006)$ after hemin treatment $(100 \mu \mathrm{M})$ relative to levels in untreated cells, indicating increased autophagy (Fig. 2a and c). Furthermore, TEM analysis revealed increased formation of autophagosomes after hemin treatment (Fig. 2d).

\section{GM130 overexpression reduces autophagosome formation and rescues tight junction integrity andGolgi morphology in Bend.3 cells}

To confirm the role of GM130 in autophagy and tight junction integrity, we transfected Bend. 3 cells with either an empty pcDNA3.1 vector or pcDNA3.1-GM130. After a $12 \mathrm{~h}$ of incubation, cells were treated with hemin for $48 \mathrm{~h}$. After confirming GM130 overexpression in both vehicle- and hemin-treated Bend. 3 cells (Fig. 3a and c), we found that GM130 overexpression inhibited autophagosome formation by decreasing LC3b levels $(p<0.05)$ and increasing p62 levels $(p<0.05)$ (Fig. 3a and b). Additionally, we observed improved occludin $(p<0.05)$ and ZO-1 ( $<0.05)$ expression after GM130 overexpression (Fig. 3a and b). Furthermore, GM130 overexpression repaired tight junction integrity in hemin-treated Bend.3 cells (Fig. 3c and d), and IF staining showed that tight junction proteins were more continuous relative to cells transfected with the empty vector (Fig. 3e and f). Moreover, we observed that LC3-II levels decreased significantly $(p<0.05)$ and p62 levels increased $(p<0.05)$ in hemin-treated GM130-overexpressing cells relative to those transfected with the empty vector (Fig. $3 c$ and d), along with downregulated autophagosome formation as revealed through TEM (Fig. 2d). These findings indicated that GM130 overexpression reduced hemin-induced autophagosome formation and repaired tight junction integrity and Golgi morphology in Bend.3 cells.

\section{GM130 silencing induces autophagy formation and tight junction disruption in Bend.3 cells}


We then examined the silencing efficiency of two different siRNA constructs against GM130. si- GM130-1 significantly decreased GM130 protein levels relative to siRNA-NC $(p<0.05)$ and was selected for further analysis (Fig. 4a). Additionally, we observed no obvious difference in cell viability between siRNA-NC and siGM130-1 or siGM130-2 groups ( $p<0.05$; Supplementary table 1). IF analysis of GM130 to assess Golgi structure revealed variance in the Golgi apparatus from a compact, perinuclear distribution to a dispersed distribution throughout the cytoplasm after siGM130-1 transfection (Fig. 3e). These results indicated that GM130 plays an important role in maintaining Golgi structure.

To determine whether GM130 silencing plays a regulatory role in autophagy and tight junction integrity, we evaluated changes in LC3 and tight junction protein levels using WB. siGM130 transfection significantly increased LC3-II levels $(p<0.05)$ and decreased p62 levels $(p<0.05)$ relative to those in siRNA-NC-transfected cells (Fig. $4 c$ and d). Moreover, TEM analysis confirmed an increase in autophagosome formation in the cytoplasm of GM130-silenced cells (Fig. 4b). Furthermore, siGM130 significantly decreased the levels of tight junction proteins, including occludin $(p<0.05)$ and ZO-1 $(p<$ 0.05), and resulted in BBB disruption (Fig. $\mathbf{4 C}$ and d). IF revealed increased tight junction discontinuity (Fig. 3e). Notably, we found that hemin administration triggered GM130-mediated Golgi stress and resulted in altered Golgi morphology and BBB damage, which mimicked the effects of GM130 silencing.

\section{Disrupted tight junction integrity induced by GM130 silencing is partially a consequence ofexcessive activation of autophagy in Bend. 3 cells}

As noted, GM130 silencing induced autophagosome formation and BBB disruption. To further investigate whether the mechanism underlying GM130-silenced alterations in tight junction status are mediated by excessive activation of autophagy, we administered the autophagy inhibitor 3-MA to siGM130-transfected cells and evaluated changes in protein levels. We found that LC3-II levels significantly decreased $(p<$ $0.05)$ and p62 levels increased $(p<0.05)$ in 3-MA-treated siGM130-transfected cells $(p<0.05)$ (Fig. 4e and $f)$, while both occludin $(p<0.05)$ and ZO-1 $(p<0.05)$ levels were elevated in these cells relative to those in cells only transfected with siGM130 (Fig. 4 e and f). These results indicated that 3-MA treatment partially restored occludin and ZO-1 levels, suggesting that disruption of tight junctions induced by GM130 silencing might be a consequence of excessive activation of autophagy.

\section{GM130 overexpression reduces BBB permeability, alleviates brain edema, and improvesneurologic outcomes after ICH in vivo}

To determine the effect of GM130 silencing on BBB integrity and neurological function after $\mathrm{ICH}$, siGM130-1 or siRNA-NC was transfected via injection into the lateral ventricles of rats using a stereotactic 
technique prior to establishment of the ICH model. At 24-h post-transfection, collagenase was administered into the basal ganglia of rats to establish the in vivo ICH model. All of the following tests were performed $48 \mathrm{~h}$ after establishing the ICH model. Figure $\mathbf{5 c}$ shows that the Garcia scores were lower in siGM130-transfected ICH rats relative to siRNA-NC-transfected rats $(p<0.001)$, indicating that GM130 silencing further aggravated neurological defects. Additionally, EB extravasation assay (Fig. 5a and b) and determination of brain water content (Fig. $5 d)$ revealed that vascular leakage $(p<0.001)$ and the dry-wet weight ratio $(p<0.001)$ in GM130-silenced ICH rats were significantly higher than those in siRNANC-transfected rats.

Similarly, to explore the effect of GM130 overexpression on the BBB and neurological function after ICH, AAV-GM130 overexpression was induced in rats before ICH establishment via injection of AAV-GM130 or AAV-Control into the lateral ventricle using a stereotactic technique. After 1 month, collagenase was administered into the basal ganglia of rats to establish the in vivo ICH model, with subsequent tests performed $48 \mathrm{~h}$ after model establishment. Contrary to the effects of GM130 silencing, GM130 overexpression significantly improved the Garcia scores in ICH rats relative to those in AAV-Controltransfected rats $(p<0.001 ; \mathbf{F i g} . \mathbf{5 g})$. Moreover, EB extravasation assay and the brain water content revealed less vascular leakage (Fig. 5e and f) and a lower dry-wet weight ratio (Fig. 5h) in the GM130overexpressing ICH rats $(p<0.001)$. These findings suggested that GM130 overexpression exerted a neuroprotective effect in the $\mathrm{ICH}$ rat model by reducing BBB permeability and alleviating neurological defects and brain edema.

\section{GM130 overexpression exerts a neuroprotective effect by rescuing the expression of tight junction proteins in $\mathrm{ICH}$ in vivo}

We found that attenuated GM130 expression resulted in reduced tight junction integrity and significantly increased autophagy over time in the ICH models (from 12-72 h; $p<0.05$; Fig. 6a and b). Therefore, we chose $48 \mathrm{~h}$ as the time point for collecting brain tissue after ICH. To explore whether GM130 overexpression alleviates tight junction disruption and reduces excessive autophagy activation, we performed WB analysis of samples collected from the hematoma in $\mathrm{ICH}$ rats that were transfected with either AAV-GM130 or AAV-Control. After verifying successful overexpression of GM130 in the rat brain (Fig. 6e), WB analysis of samples showed significant increases in levels of tight junction proteins (ZO-1 and occludin) compared to those of tight junction proteins in AAV-Control-transfected rats ( $<0.01$; Fig. $6 \mathbf{c}$ and d). Moreover, double IF labeling of GM130 and occludin revealed that occludin was more widely distributed in GM130-overexpressing ICH rats (Fig. 7). Further analysis of the underlying mechanism associated with the rescued expression of tight junction proteins revealed attenuated LC3-II levels and increased p62 levels in GM130-overexpressing ICH rats ( $p<0.01$; Fig. $6 \mathrm{c}$ and d). Furthermore, IF analysis showed a smaller distribution of autophagosomes in these rats compared to that in AAV-Controltransfected rats (Fig. 8a). 


\section{The decreased expression of tight junction proteins induced by GM130 silencing is partiallyalleviated by autophagy inhibition in $\mathrm{ICH}$ in vivo}

siGM130-1 significantly decreased GM130 protein levels compared to siRNA-NC $(p<0.05)$ and was chosen for further analysis (Fig. 9c). In contrast to the neuroprotective effect of GM130 overexpression, GM130 silencing promoted the formation of autophagosomes (Fig.8b) and impaired tight junction protein expression in hemorrhaged rat brains (Fig.9 a, b, and Fig.10). To determine the role of autophagy in the observed tight junction dysfunction induced by GM130 silencing, we administered 3-MA to siGM130- transfected ICH model rats and evaluated changes in protein levels through WB. After verifying GM130 silencing and concomitant downregulation of occludin and Z0-1, we found that 3-MA treatment partially rescued occludin and ZO-1 levels (Fig. 9a and b). These findings suggested that autophagy activation plays a role in the attenuated expression of tight junction proteins induced by GM130 silencing.

\section{Discussion}

Therapies targeting protection of BBB integrity can reduce neurological defects induced by ICH [3]. In this study, we explored the role of GM130 in BBB protection and found that regulation of GM130 protected BBB integrity by maintaining autophagic flux at an appropriate level and alleviating neurological defects following $\mathrm{ICH}$.

The BBB acts as a physical and physiological barrier that protects the brain from toxic substances. BBB damage results in brain edema and prompts an inflammatory response. Herein, we found that tight junction proteins were further damaged during prolonged $\mathrm{ICH}$, which was accompanied by destruction of the BBB and onset of brain edema and neurological dysfunction at 48-h after establishment of the ICH model, as reported in previous studies [33]. Many signaling factors, such as matrix metalloproteinase-9 [34] and vascular endothelial growth factor [35], can control BBB permeability by regulating its structural components. Moreover, activation of the autophagy signaling pathway also regulates the BBB [36].

Autophagy is a tightly regulated process that targets bulk removal of degraded cytoplasmic macromolecules and organelles in mammalian cells via lysosomes in order to maintain cell homeostasis [37]. A previous study demonstrated that higher numbers of autophagic neurons in patients with $\mathrm{ICH}$ correlated with increased severity of neuronal dysfunction [38]. In the present study, elevated LC3-II and decreased p62 levels in the $\mathrm{ICH}$ rat brain indicated excessive autophagic flux induced by brain injury. Moreover, the negative regulatory effect on tight junction proteins induced by GM130 silencing in Bend.3 cells could be reversed by administering an autophagy inhibitor. These findings suggest that inhibition of autophagosome formation can reduce BBB injury induced by excessive autophagy.

Autophagy can be influenced by Golgi dysfunction. As a signaling platform, the Golgi apparatus provides not only a membrane for autophagosome formation but also a location for autophagosome induction 
and elongation [15]. Additionally, Golgi-related proteins directly participate in autophagosome formation [15]. In our ICH in vivo models, LC3-II expression around the hematoma in the GM130-overexpression group was lower than that in the NC group. Moreover, the level of perihematomal LC3-II in the GM130silenced group was higher than that in the NC group, with similar results under both conditions observed in $\mathrm{ICH}$ in vivo models. These results indicated that GM130 exerted a negative regulatory effect on autophagy in both in vivo and in vitro IHC models, which agrees with the findings of a previous study [20].

We observed Golgi fragmentation and decreased GM130 levels in the brains of ICH rats, both of which are associated with Golgi stress [23]. Golgi dysfunction derived from Golgi stress might be an essential process in disruption of several cell signaling transduction pathways, including the autophagy signaling pathway [39]. Based on these results, we hypothesize that excessive autophagy signaling induced by decreased GM130 levels further disrupts BBB integrity and promotes brain edema in $\mathrm{ICH}$. Our findings demonstrated that tight junction disruption and autophagic flux increased in the GM130-silenced ICH group relative to the vehicle-control ICH group. Moreover, further mechanistic study in vitro showed higher levels of occludin and ZO-1 in 3-MA-treated cells than those in control cells, demonstrating that 3-MA could partially restore expression of tight junction proteins and the accompanying damage induced by GM130 silencing in Bend. 3 cells. These results support our hypothesis. Furthermore, GM130overexpression ICH rat models showed higher levels of tight junction proteins, less intense EB staining, and lower perihematomal brain water content than that of rats receiving cells transfected with AAVControl. These findings suggest that targeted upregulation of GM130 might represent a potential therapeutic strategy for $\mathrm{ICH}$.

In summary, these results indicate that GM130 overexpression is beneficial for promoting tight junction integrity and repair and improving neurobehavioral recovery in $\mathrm{ICH}$ rats by directly modulating its downstream targets in the autophagy pathway. The data suggest GM130 as a possible therapeutic target for acute brain injury after $\mathrm{ICH}$.

\section{Declarations}

\section{Funding}

This work was funded by the National Natural Science Foundation of China (\#81571181).

\section{Conflicts of interest}

The authors have no relevant financial or non-financial interests to disclose.

\section{Availability of data and material}

Data will be made available on reasonable request.

\section{Code availability}


Not applicable

\section{Authors' contributions}

Shuwen Deng performed the experiments and drafted the manuscript; Qing Hu and Qiang He analyzed the data; Xiqian Chen helped draft the manuscript; Wei Lu conceived and designed the study; and all authors read and approved the final manuscript

\section{Ethics approval}

The study was conducted according to the guidelines for the care and use of laboratory animals of Central South University, and the experiment protocols were approved by the Central South University Animal Care and Use Committee (approval number: 2021013).

\section{Consent to participate}

Not applicable

\section{Consent for publication}

Not applicable

\section{Compliance with ethical standards}

Disclosure of potential conflicts of interest

The authors have no conflicts of interest to declare that are relevant to the content of this article.

Research involving human participants and/or animals

The study was conducted according to the guidelines for the care and use of laboratory animals of Central South University, and the experiment protocols were approved by the Central South University Animal Care and Use Committee (approval number: 2021013).

\section{Informed consent}

Not applicable

\section{References}

1. Zeng, J., et al., Isoliquiritigenin alleviates early brain injury after experimental intracerebral hemorrhage via suppressing ROS- and/or NF-KB-mediated NLRP3 inflammasome activation by promoting Nrf2 antioxidant pathway. Journal of Neuroinflammation, 2017. 14(1).

2. Zhu, H., et al., Role and mechanisms of cytokines in the secondary brain injury after intracerebral hemorrhage. Prog Neurobiol, 2019. 178: p. 101610. 
3. Duan, X., et al., Intracerebral Hemorrhage, Oxidative Stress, and Antioxidant Therapy. Oxid Med Cell Longev, 2016. 2016: p. 1203285.

4. Keep, R.F., et al., Blood-brain barrier function in intracerebral hemorrhage. Acta Neurochir Suppl, 2008. 105: p. 73-7.

5. Wang, Z., et al., Melatonin Alleviates Intracerebral Hemorrhage-Induced Secondary Brain Injury in Rats via Suppressing Apoptosis, Inflammation, Oxidative Stress, DNA Damage, and Mitochondria Injury. Transl Stroke Res, 2018. 9(1): p. 74-91.

6. Keep, R.F., et al., Brain endothelial cell junctions after cerebral hemorrhage: Changes, mechanisms and therapeutic targets. J Cereb Blood Flow Metab, 2018. 38(8): p. 1255-1275.

7. Sajja, R.K., S. Rahman, and L. Cucullo, Drugs of abuse and blood-brain barrier endothelial dysfunction: A focus on the role of oxidative stress. J Cereb Blood Flow Metab, 2016. 36(3): p. 53954.

8. Kim, K.A., et al., Role of Autophagy in Endothelial Damage and Blood-Brain Barrier Disruption in Ischemic Stroke. Stroke, 2018. 49(6): p. 1571-1579.

9. Wang, P., et al., Autophagy in ischemic stroke. Prog Neurobiol, 2018. 163-164: p. 98-117.

10. Yang, Z., et al., Autophagy Protects the Blood-Brain Barrier Through Regulating the Dynamic of Claudin-5 in Short-Term Starvation. Front Physiol, 2019. 10: p. 2.

11. Song, F., et al., Therapeutic time window and regulation of autophagy by mild hypothermia after intracerebral hemorrhage in rats. Brain Res, 2018. 1690: p. 12-22.

12. Zhao, H., et al., Role of autophagy in early brain injury after subarachnoid hemorrhage in rats. Mol Biol Rep, 2013. 40(2): p. 819-27.

13. Filomeni, G., D. De Zio, and F. Cecconi, Oxidative stress and autophagy: the clash between damage and metabolic needs. Cell Death Differ, 2015. 22(3): p. 377-88.

14. Lin, Y., et al., Cancer and ER stress: Mutual crosstalk between autophagy, oxidative stress and inflammatory response. Biomed Pharmacother, 2019. 118: p. 109249.

15. Deng, S., et al., Golgi Apparatus: A Potential Therapeutic Target for Autophagy-Associated Neurological Diseases. Front Cell Dev Biol, 2020. 8: p. 564975.

16. Barthet, V.J.A. and K.M. Ryan, Autophagy in Neurodegeneration: Can't Digest It, Spit It Out! Trends in Cell Biology, 2018. 28(3): p. 171-173.

17. Bravo-San Pedro, J.M., G. Kroemer, and L. Galluzzi, Autophagy and Mitophagy in Cardiovascular Disease. Circ Res, 2017. 120(11): p. 1812-1824.

18. Saha, S., et al., Autophagy in health and disease: A comprehensive review. Biomed Pharmacother, 2018. 104: p. 485-495.

19. Antunes, F., et al., Autophagy and intermittent fasting: the connection for cancer therapy? Clinics (Sao Paulo), 2018. 73(suppl 1): p. e814s.

20. Chang, S.H., et al., GOLGA2/GM130, cis-Golgi matrix protein, is a novel target of anticancer gene therapy. Mol Ther, 2012. 20(11): p. 2052-63. 
21. Joachim, J., et al., Activation of ULK Kinase and Autophagy by GABARAP Trafficking from the Centrosome Is Regulated by WAC and GM130. Mol Cell, 2015. 60(6): p. 899-913.

22. Joachim, J. and S.A. Tooze, GABARAP activates ULK1 and traffics from the centrosome dependent on Golgi partners WAC and GOLGA2/GM130. Autophagy, 2016. 12(5): p. 892-3.

23. Qiu, K., et al., [Effect of butylphthalide on blood-brain barrier after cerebral hemorrhage in SD rats and the mechanisms]. Zhong Nan Da Xue Xue Bao Yi Xue Ban, 2019. 44(12): p. 1321-1329.

24. He, Q., et al., Herpes Simplex Virus 1-Induced Blood-Brain Barrier Damage Involves Apoptosis Associated With GM130-Mediated Golgi Stress. Front Mol Neurosci, 2020. 13: p. 2.

25. Li, Z., et al., Inhibition of IncRNA XIST Improves Myocardial I/R Injury by Targeting miR-133a through Inhibition of Autophagy and Regulation of SOCS2. Mol Ther Nucleic Acids, 2019. 18: p. 764-773.

26. $\mathrm{Xi}$, $\mathrm{T}$., et al., miR-27a-3p protects against blood-brain barrier disruption and brain injury after intracerebral hemorrhage by targeting endothelial aquaporin-11. J Biol Chem, 2018. 293(52): p. 20041-20050.

27. Chen, Y., et al., Norrin protected blood-brain barrier via frizzled-4/ $\beta$-catenin pathway after subarachnoid hemorrhage in rats. Stroke, 2015. 46(2): p. 529-36.

28. Zhu, Q., et al., Aggf1 attenuates neuroinflammation and BBB disruption via PI3K/Akt/NF-KB pathway after subarachnoid hemorrhage in rats. J Neuroinflammation, 2018. 15(1): p. 178.

29. Garcia, J.H., et al., Neurological deficit and extent of neuronal necrosis attributable to middle cerebral artery occlusion in rats. Statistical validation. Stroke, 1995. 26(4): p. 627-34; discussion 635.

30. Wang, S., Z. Zhang, and Q. Gao, Transfer of microRNA-25 by colorectal cancer cell-derived extracellular vesicles facilitates colorectal cancer development and metastasis. Mol Ther Nucleic Acids, 2021. 23: p. 552-564.

31. Zhao, H., et al., Effects of Simulated Microgravity on Ultrastructure and Apoptosis of Choroidal Vascular Endothelial Cells. Front Physiol, 2020. 11: p. 577325.

32. Lei, B., et al., miR-615-3p promotes the epithelial-mesenchymal transition and metastasis of breast cancer by targeting PICK1/TGFBRI axis. J Exp Clin Cancer Res, 2020. 39(1): p. 71.

33. Zeng, Z., X. Gong, and Z. Hu, L-3-n-butylphthalide attenuates inflammation response and brain edema in rat intracerebral hemorrhage model. Aging (Albany NY), 2020. 12(12): p. 11768-11780.

34. Wang, R., et al., Porcine reproductive and respiratory syndrome virus induces HMGB1 secretion via activating PKC-delta to trigger inflammatory response. Virology, 2018. 518: p. 172-183.

35. Almutairi, M.M., et al., Factors controlling permeability of the blood-brain barrier. Cell Mol Life Sci, 2016. 73(1): p. 57-77.

36. Zhang, S., et al., Autophagy- and MMP-2/9-mediated Reduction and Redistribution of ZO-1 Contribute to Hyperglycemia-increased Blood-Brain Barrier Permeability During Early Reperfusion in Stroke. Neuroscience, 2018. 377: p. 126-137.

37. Huang, G., et al., Autophagy is an important action mode for functionalized selenium nanoparticles to exhibit anti-colorectal cancer activity. Biomater Sci, 2018. 6(9): p. 2508-2517. 
38. Wu, C., et al., Increased perihematomal neuron autophagy and plasma thrombin-antithrombin levels in patients with intracerebral hemorrhage: An observational study. Medicine (Baltimore), 2019. 98(39): p. e17130.

39. Martínez-Menárguez, J., et al., Golgi Fragmentation in Neurodegenerative Diseases: Is There a Common Cause? Cells, 2019. 8(7).

\section{Tables}

Table 1. Garcia neurological score

\begin{tabular}{|c|c|c|c|c|}
\hline \multirow[t]{2}{*}{ Test } & \multicolumn{4}{|l|}{ Score } \\
\hline & 0 & 1 & 2 & 3 \\
\hline $\begin{array}{l}\text { Spontaneous } \\
\text { activity (in cage for } \\
5 \text { min) }\end{array}$ & No movement & Barely moves & $\begin{array}{l}\text { Moves but does not } \\
\text { approach at least } \\
\text { three sides of cage }\end{array}$ & $\begin{array}{l}\text { Moves and } \\
\text { approaches at } \\
\text { least three sides } \\
\text { of cage }\end{array}$ \\
\hline $\begin{array}{l}\text { Symmetry of } \\
\text { movement (four } \\
\text { limbs) }\end{array}$ & $\begin{array}{l}\text { Left side: no } \\
\text { movement }\end{array}$ & $\begin{array}{l}\text { Left side: } \\
\text { slight } \\
\text { movement }\end{array}$ & $\begin{array}{l}\text { Left side: moves } \\
\text { slowly }\end{array}$ & $\begin{array}{l}\text { Both sides: move } \\
\text { symmetrically }\end{array}$ \\
\hline $\begin{array}{l}\text { Symmetry of } \\
\text { forelimbs } \\
\text { (outstretching while } \\
\text { held by tail) }\end{array}$ & $\begin{array}{l}\text { Left side: no } \\
\text { movement, } \\
\text { no } \\
\text { outreaching }\end{array}$ & $\begin{array}{l}\text { Left side: } \\
\text { slight } \\
\text { movement to } \\
\text { outreach }\end{array}$ & $\begin{array}{l}\text { Left side: moves and } \\
\text { outreaches less than } \\
\text { right side }\end{array}$ & $\begin{array}{l}\text { Symmetrical } \\
\text { outreach }\end{array}$ \\
\hline $\begin{array}{l}\text { Climbing wall of } \\
\text { wire cage }\end{array}$ & - & Fails to climb & Left side is weak & Normal climbing \\
\hline $\begin{array}{l}\text { Reaction to touch } \\
\text { on either side of } \\
\text { trunk }\end{array}$ & - & $\begin{array}{l}\text { No response } \\
\text { on left side }\end{array}$ & $\begin{array}{l}\text { Weak response on } \\
\text { left side }\end{array}$ & $\begin{array}{l}\text { Symmetrical } \\
\text { response }\end{array}$ \\
\hline $\begin{array}{l}\text { Response to } \\
\text { vibrissae touch }\end{array}$ & - & $\begin{array}{l}\text { No response } \\
\text { on left side }\end{array}$ & $\begin{array}{l}\text { Weak response on } \\
\text { left side }\end{array}$ & $\begin{array}{l}\text { Symmetrical } \\
\text { response }\end{array}$ \\
\hline
\end{tabular}

\section{Figures}


a

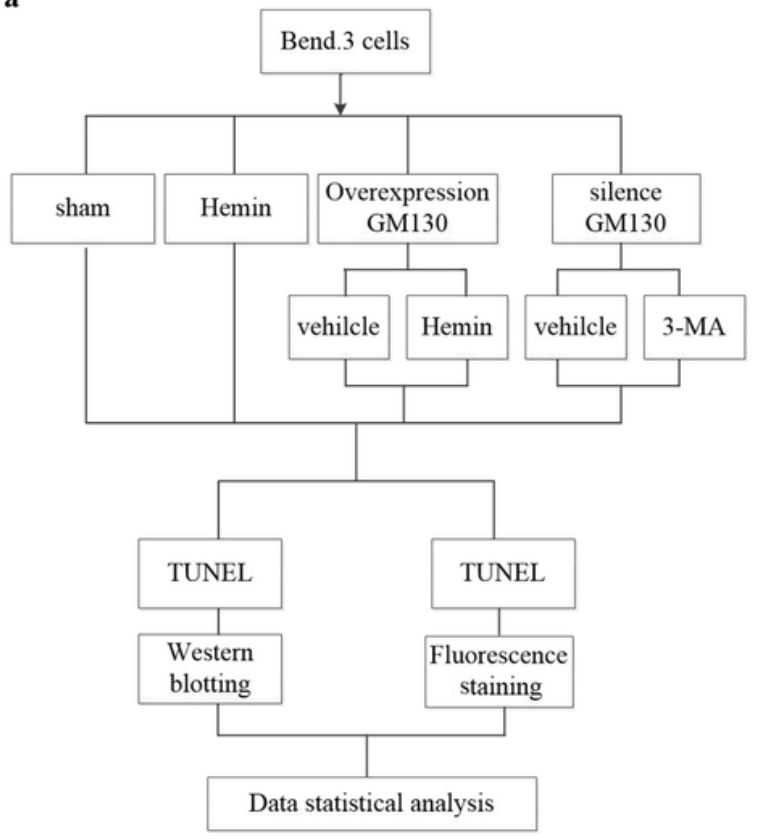

b

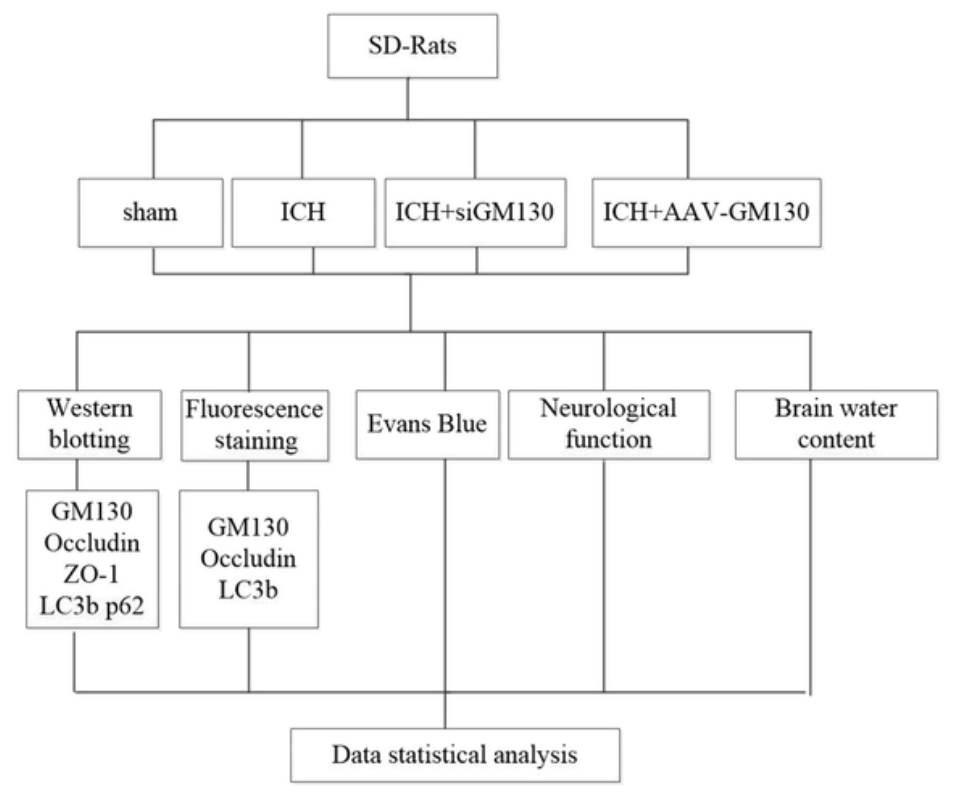

\section{Figure 1}

Experimental design. a. Experiment 1 was designed to investigate the effect of GM130 silencing or overexpression on the BBB in vitro. b. Experiment 2 was designed to investigate the effect of GM130 silencing or overexpression on the BBB in vivo. 
a

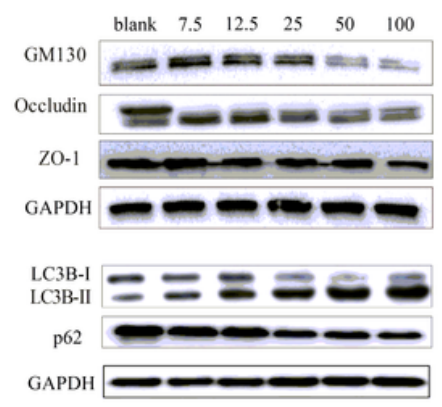

c

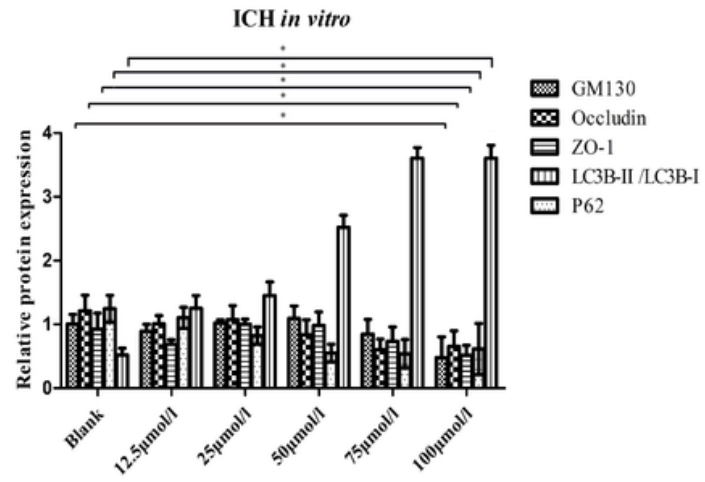

b

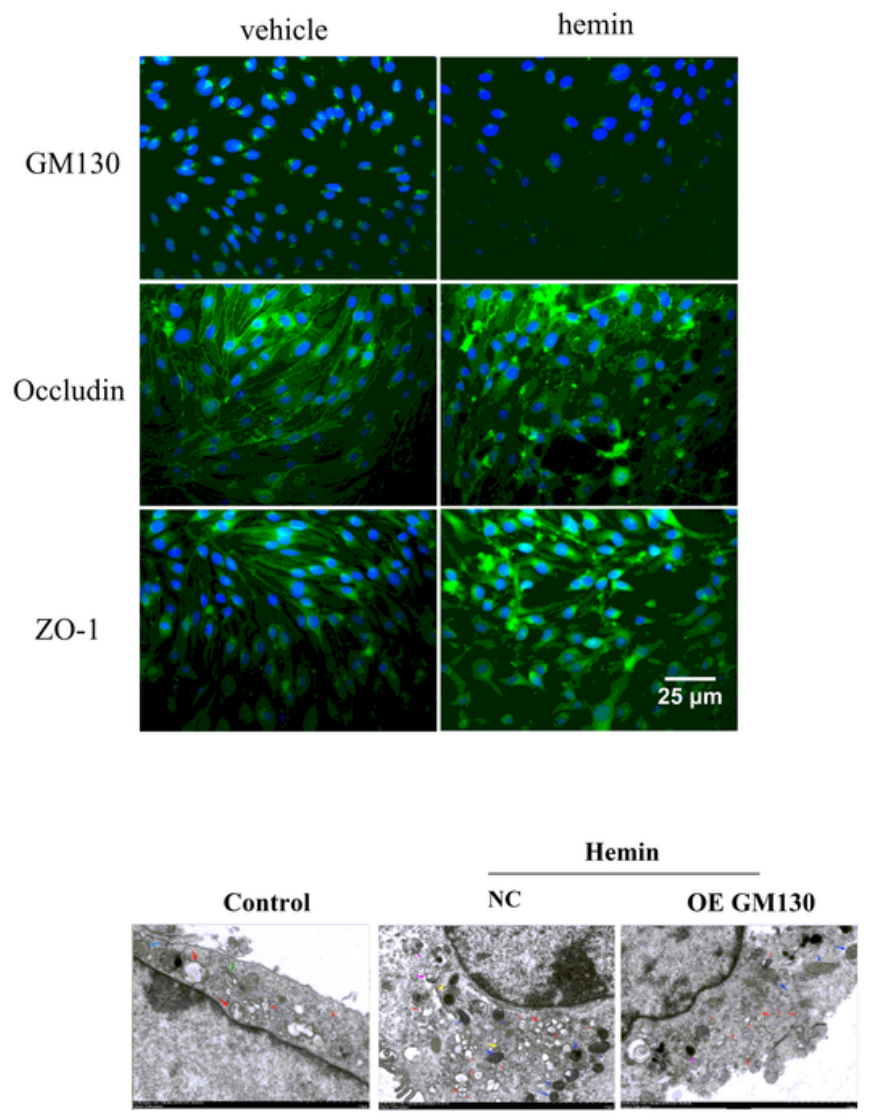

\section{Figure 2}

Hemin treatment downregulates GM130, disrupts Golgi function, destroys tight junction integrity, and induces autophagy in Bend. 3 cells. a. WB showing GM130, occludin, ZO-1, LC3, and p62 expression in Bend. 3 cells of the sham group and different groups treated with several concentrations of hemin $(n=$ 3/group). b. Fluorescence imaging showing GM130, occludin, and ZO-1 expression in Bend. 3 cells at $48 \mathrm{~h}$ after treatment with hemin. Scale bar, $25 \mu \mathrm{m}$. c. Quantification data of WB are presented as the mean \pm SD. ${ }^{*} p<0.05$. $d$. Transmission electron micrograph showing autophagosome formation (red arrow) in NC and GM130-overexpressing (OE) Bend.3 cells with or without hemin. 
a

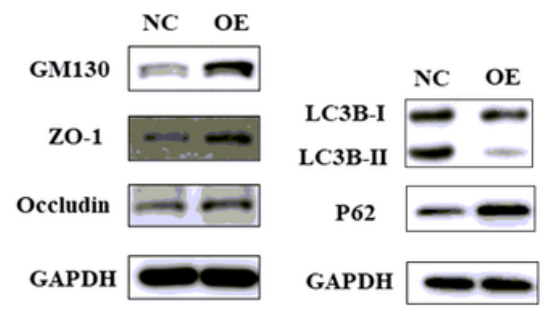

c

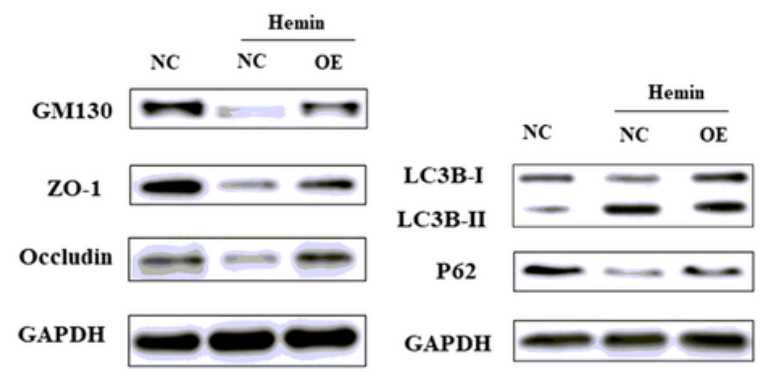

e

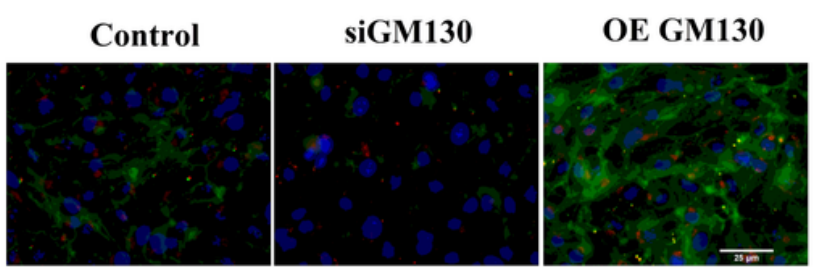

b

Overexpression of GM130 in B end.3 cell

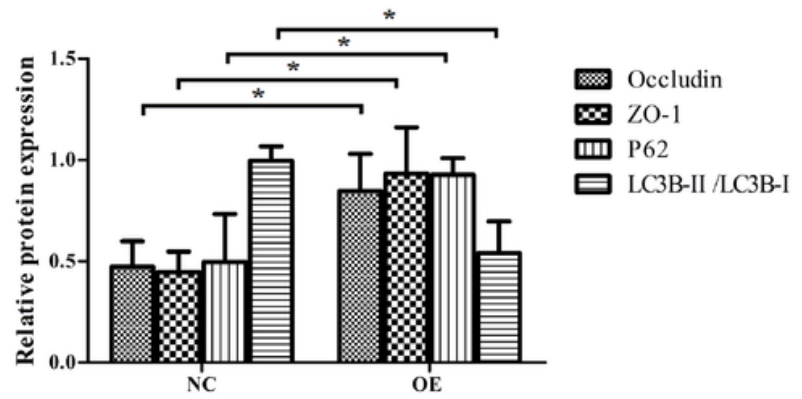

d

Overexpression of GM130 in ICH in vitro.

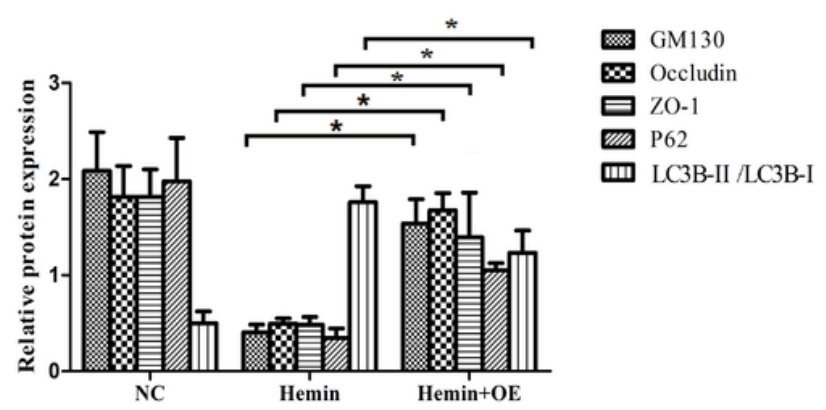

f

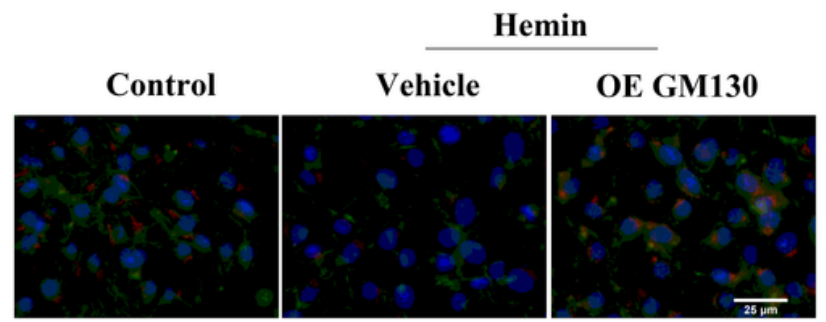

\section{Figure 3}

GOLGA2/GM130 overexpression reduces autophagosome formation and repairs tight junction integrity and Golgi morphology in Bend.3 cells. a. WB showing GM130, occludin, Z0-1, LC3, and p62 expression in NC and GM130-overexpressing Bend.3 cells ( $n=3$ /group). c. WB showing GM130, occludin, ZO-1, LC3, and p62 expression in NC Bend.3 cells and NC or GM130-overexpressing Bend.3 cells treated with hemin ( $n=3$ /group). b and d. Quantification data of WB are presented as the mean \pm SD. ${ }^{*} p<0.05$. e.

Immunostaining of GM130 in siRNA-NC, siGM130-transfected, or GM130-overexpressing (OE) Bend.3 cells. f. Immunostaining of GM130 in NC and GM130-OE Bend.3 cells with or without hemin. Scale bar, 25 $\mu \mathrm{m}$ 
a

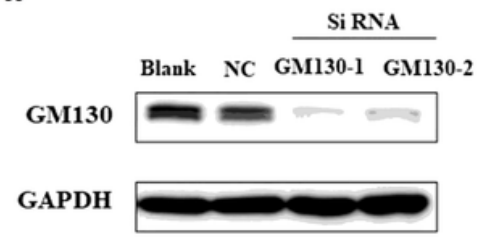

b

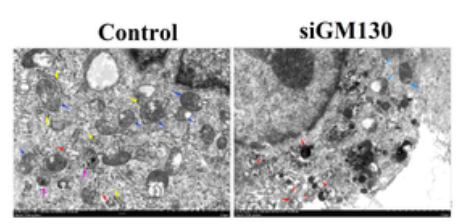

d

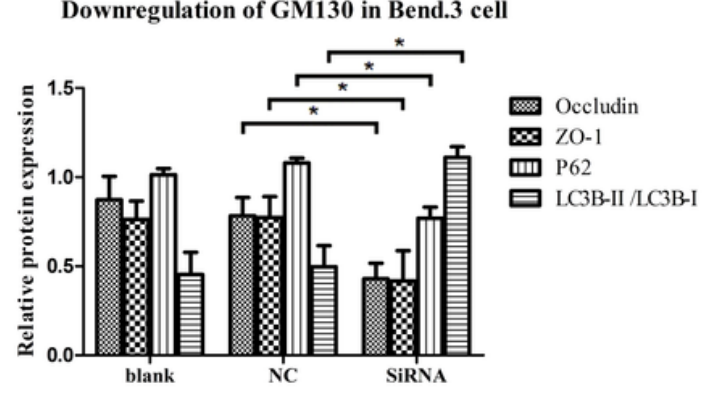

f

Downregulation of GM130 in Bend.3 cell

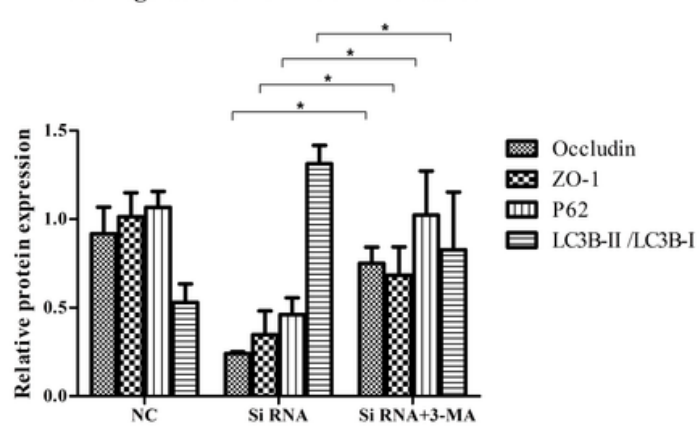

\section{Figure 4}

GOLGA2/GM130 downregulation induces autophagosome formation and tight junction disruption in Bend. 3 cells. a and c. WB showing GM130, occludin, ZO-1, LC3, and p62 expression in siRNA-NC and siGM130-transfected Bend. 3 cells ( $n=3$ /group). b. Transmission electron micrograph showing autophagosome formation (red arrow) in siRNA-NC and siGM130-transfected Bend. 3 cells. $d$ and $f$. Quantification data of WB are presented as the mean \pm SD. ${ }^{*} p<0.05$. e. WB showing GM130, occludin, ZO-1, LC3, and p62 expression in siRNA-NC and siGM130-transfected Bend.3 cells with or without 3-MA administration ( $n=3 /$ group). 
a

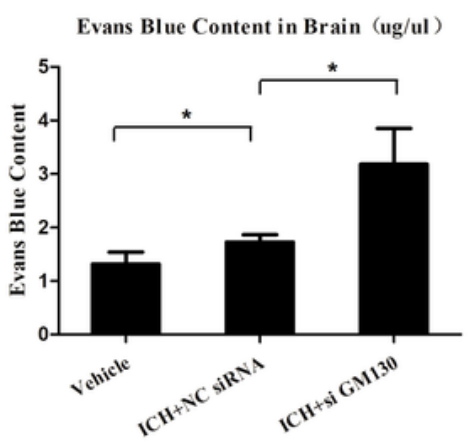

c

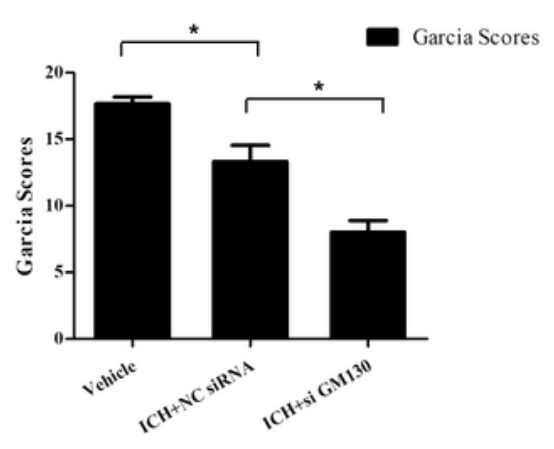

e

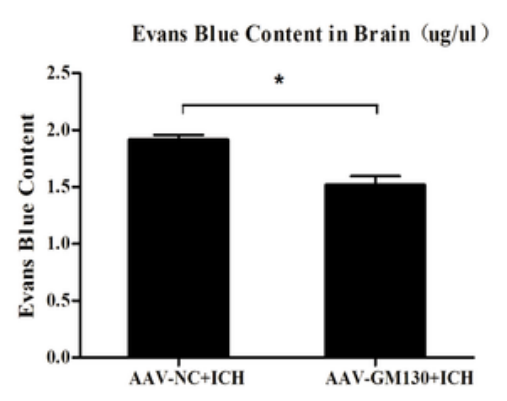

g

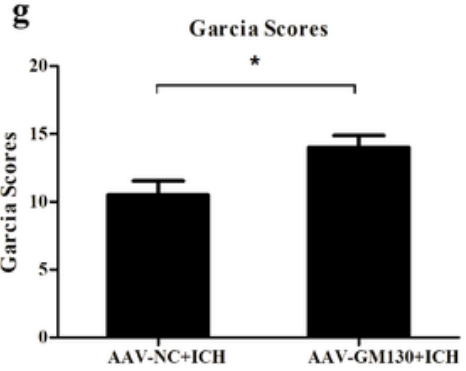

b

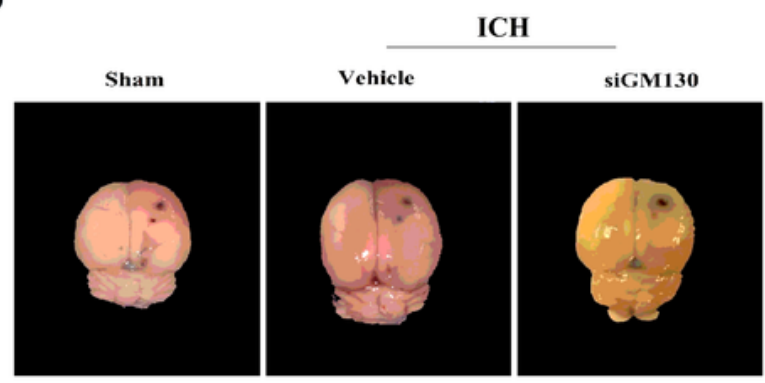

d

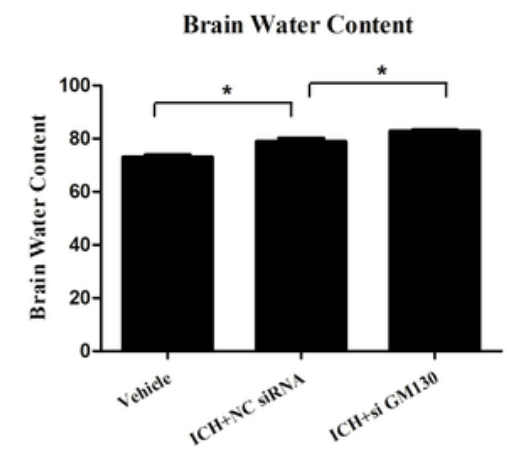

f

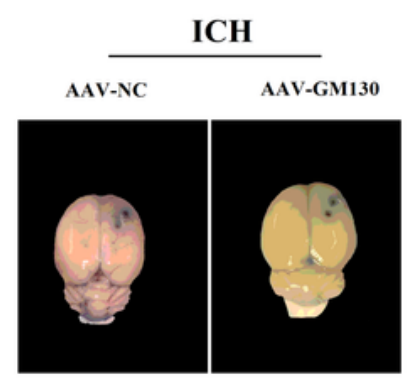

h

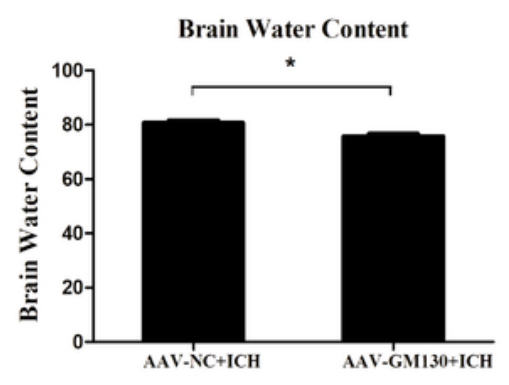

\section{Figure 5}

GM130 overexpression reduces BBB permeability, alleviates brain edema, and improves neurologic outcomes after $\mathrm{ICH}$, while GM130 silencing increases BBB disruption and aggravates brain edema and neurologic dysfunction after ICH. a. EB extravasation assay from the cerebrum of rats (shown in b) from the sham group and ICH group pretreated with or without siGM130 ( $n=3 /$ group). c. Garcia scores in sham and ICH groups pretreated with or without siGM130 ( $n=6 /$ group). $d$. Water content in sham and 
ICH groups pretreated with or without siGM130 ( $n=6 /$ group). e. EB extravasation assay from the cerebrum of rats (shown in $\mathrm{f}$ ) from the sham group and ICH group pretreated with or without AAV-GM130 ( $n=3$ /group). g. Garcia scores in sham and ICH groups pretreated with or without AAV-GM130 ( $n=$ 6 /group). h. Water content in sham and ICH groups pretreated with or without AAV-GM130 ( $n=6 /$ group). Data represent the mean \pm SD. ${ }^{*} p<0.05$

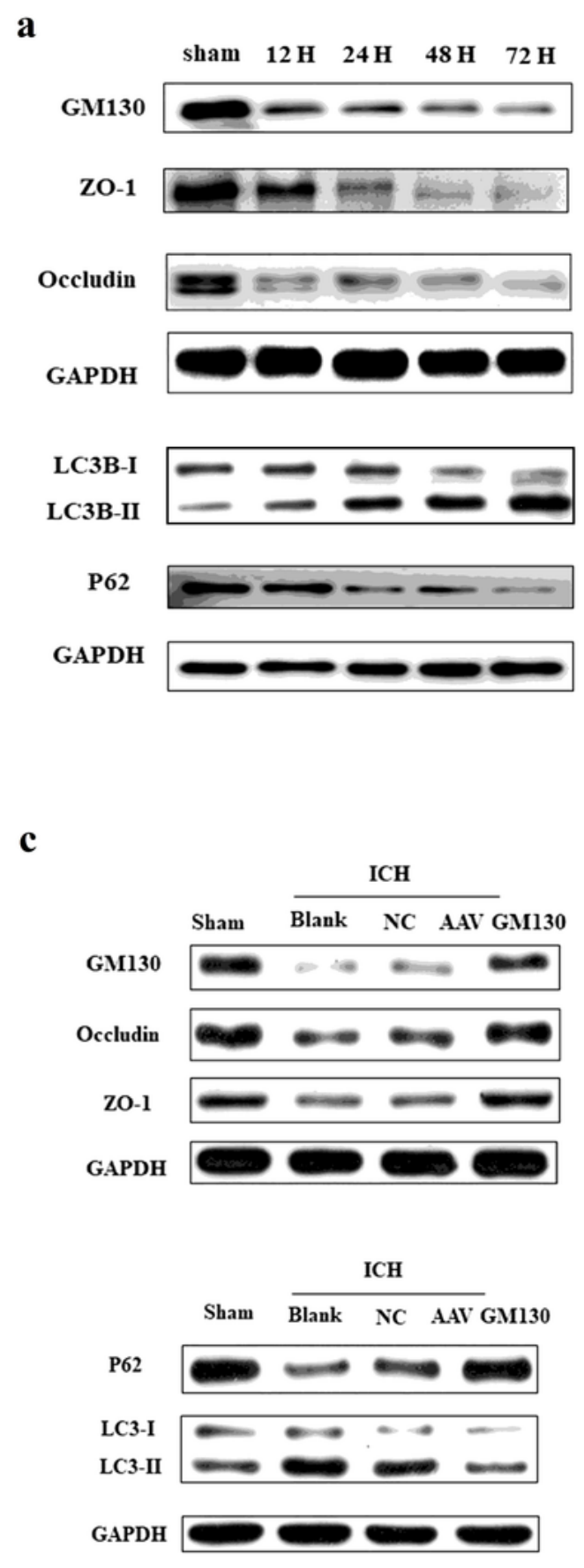

b

Relative Protein Expression in ICH

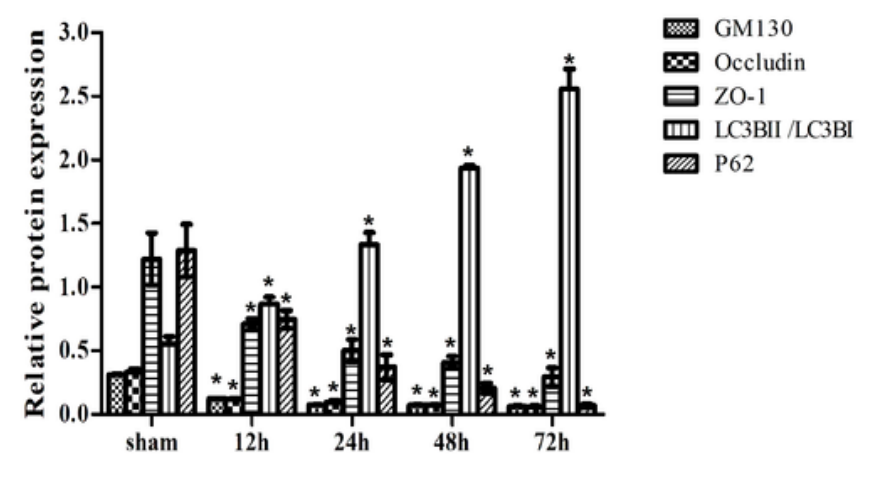

d

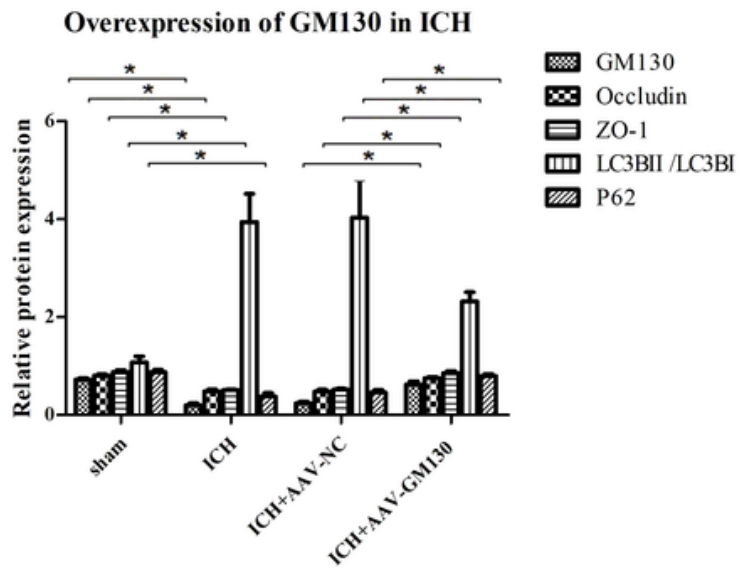

e

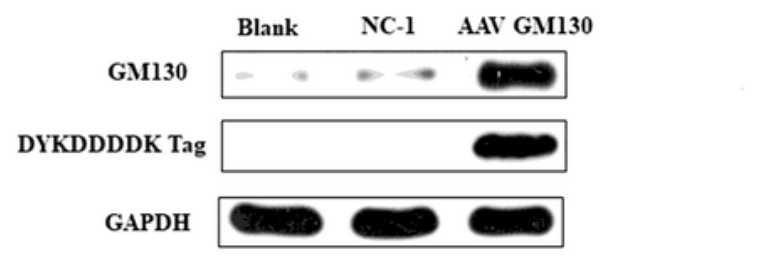

Figure 6 
Increased ICH duration decreases GM130 level and tight junction integrity and increases autophagy activation. GM130 overexpression has a neuroprotective effect on $\mathrm{ICH}$ by rescuing tight junction protein expression. a. WB showing GM130, occludin, ZO-1, LC3, and p62 expression in hemorrhaged rat brains of the sham and ICH groups after $12 \mathrm{~h}, 24 \mathrm{~h}, 48 \mathrm{~h}$, and $72 \mathrm{~h}(\mathrm{n}=6$ /group). b. Quantification data of WB are presented as the mean $\pm S D$. ${ }^{*} p<0.05$, vs. sham group. c. WB showing GM130, occludin, ZO-1, LC3, and p62 expression in hemorrhaged rat brains of the sham and ICH groups treated with saline, AAV-Control, or AAV-GM130 ( $n=6 /$ group). d. Quantification data of WB are presented as the mean $\pm S D .{ }^{*} p<0.05$. e. AAV-GM130 was successfully transfected into the rat brain.

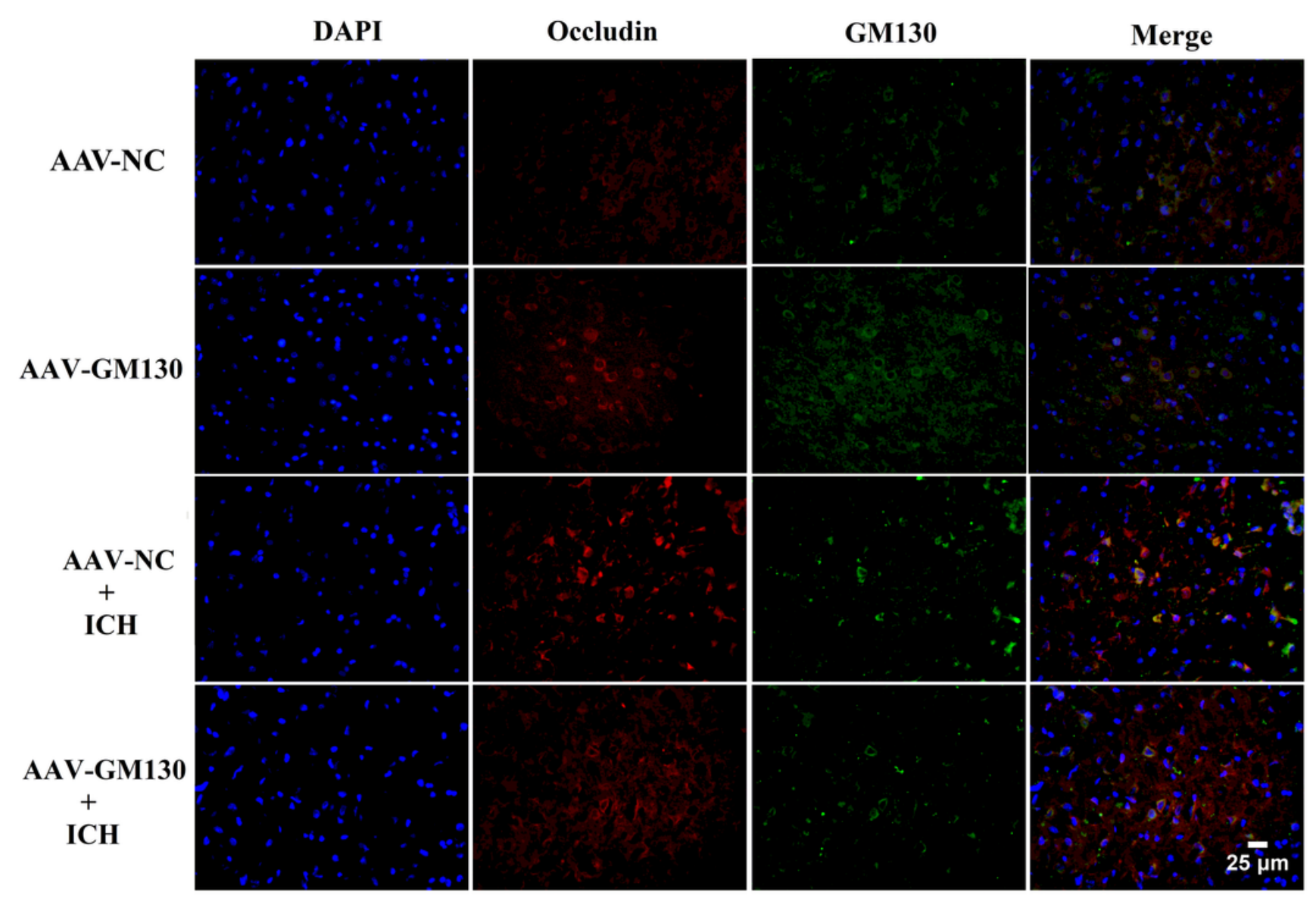

Figure 7

Immunostaining of GM130 in the hemorrhaged rat brain. Immunostaining of GM130 and occludin in hemorrhaged rat brains of the sham and ICH groups treated with saline, AAV-Control, or AAV-GM130 ( $n=$ 3/group). Scale bar, $25 \mu \mathrm{m}$ 

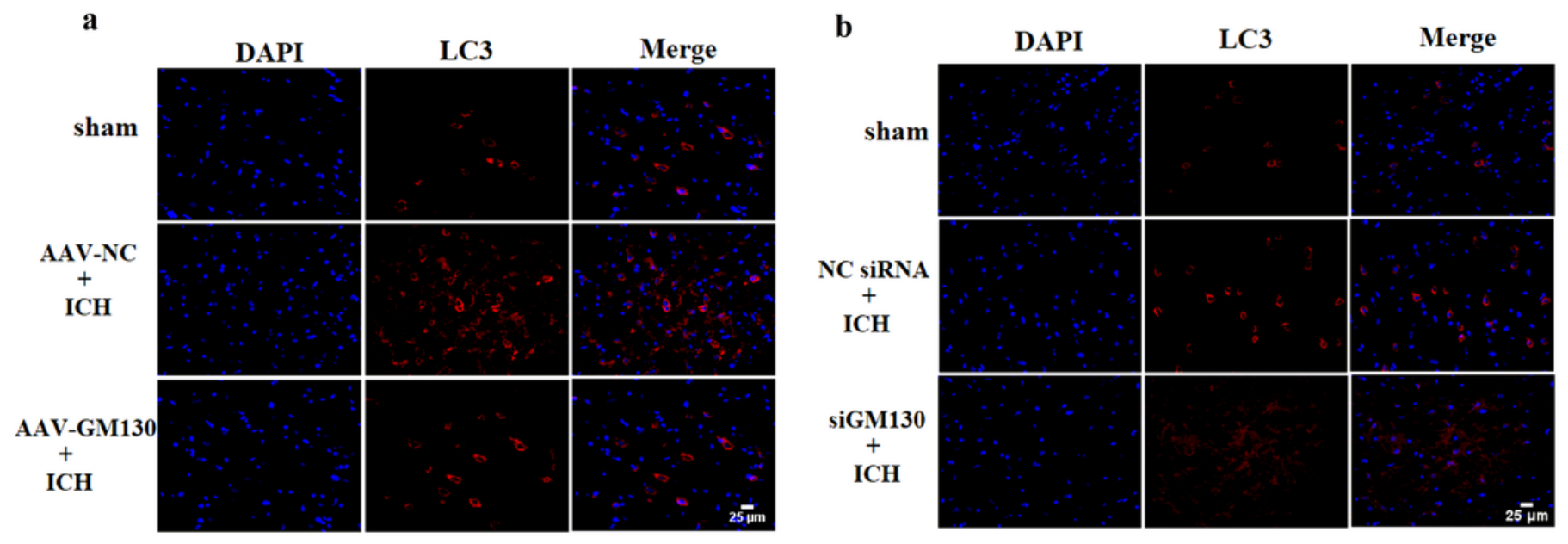

Figure 8

Immunostaining of LC3 in the hemorrhaged rat brain. a. Immunostaining of LC3 in hemorrhaged rat brains of the sham and ICH groups treated with saline, AAV-Control, or AAV-GM130 (n=3/group). b. Immunostaining of LC3 in hemorrhaged rat brains of the sham and ICH groups treated with saline, siRNA$\mathrm{NC}$, or siGM130. Scale bar, $25 \mu \mathrm{m}$

a
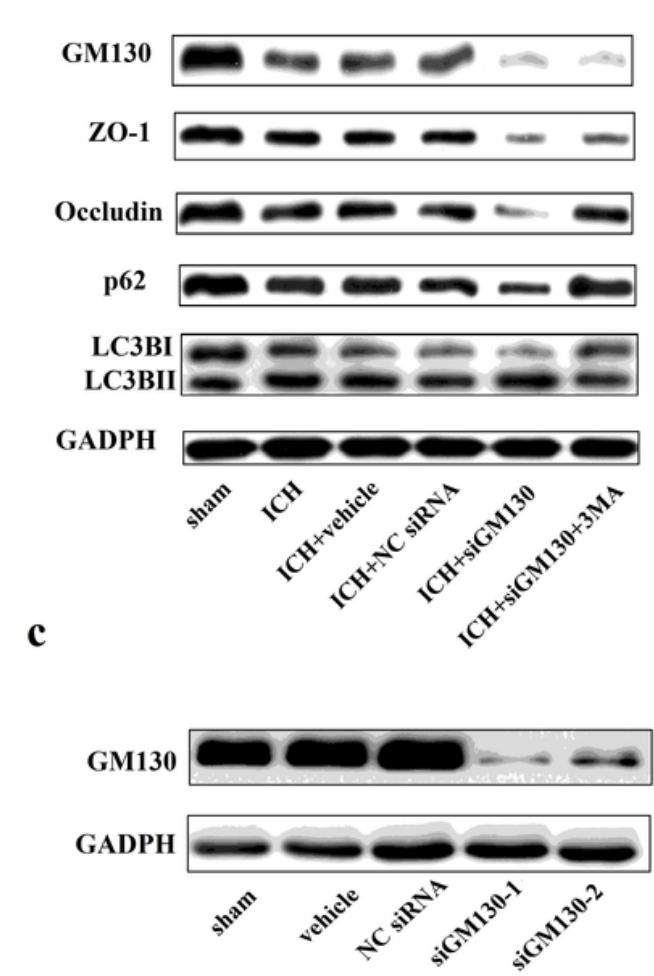

b

Downregulation of GM130 in ICH

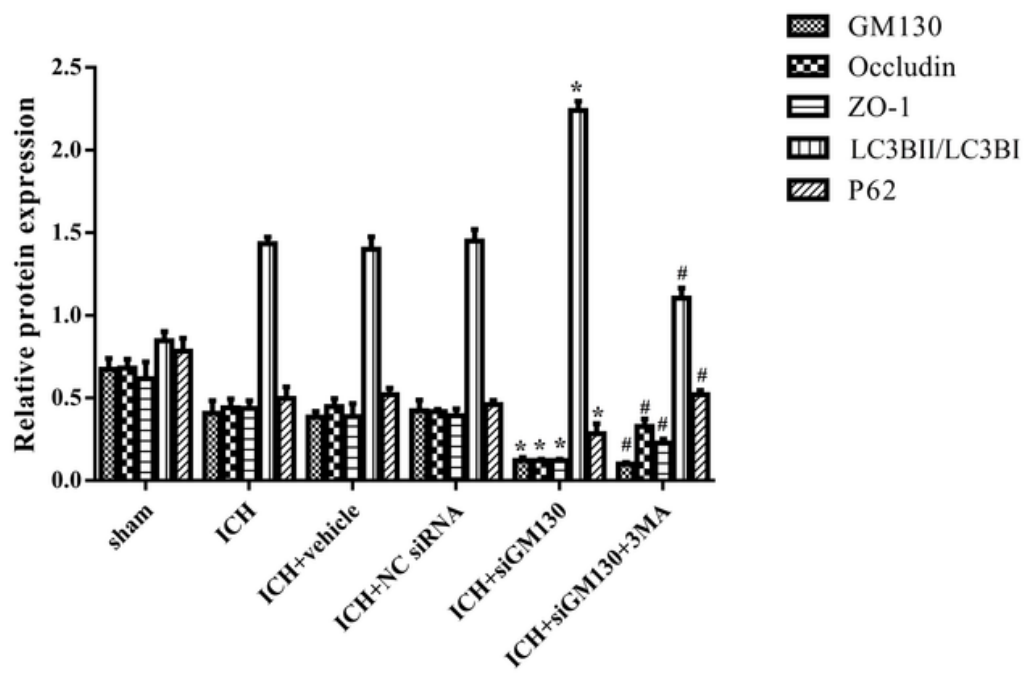

Figure 9 
GM130 silencing promotes autophagosome formation and impairs tight junction protein expression in hemorrhaged rat brains. a. WB showing GM130, occludin, ZO-1, LC3, and p62 expression in hemorrhaged rat brains of the sham and ICH groups treated with saline, siRNA-NC, siGM130, or siGM130 and 3-MA ( $\mathrm{n}=$ 6/group). b. Quantification data are presented as the mean \pm SD. ${ }^{*} p<0.05$, vs. siRNA-NC-transfected ICH group; $\# p<0.05$, vs. siGM130-transfected ICH group. c. siGM130 successfully silenced GM130 expression in the rat brain

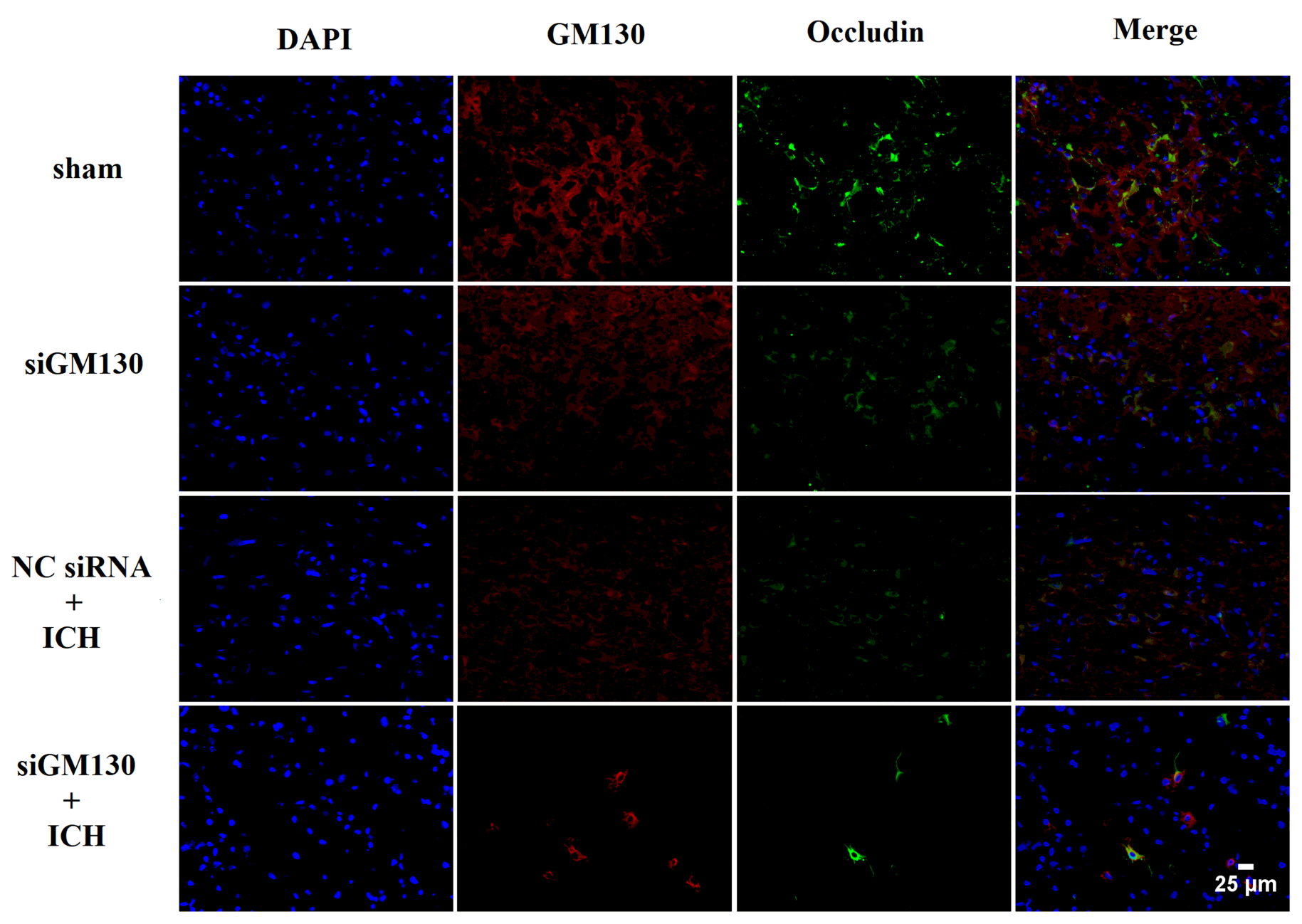

\section{Figure 10}

Immunostaining of GM130 in the hemorrhaged rat brain. Immunostaining of GM130 and occludin in hemorrhaged rat brains of the sham and ICH groups treated with saline, siRNA-NC, or siGM130 ( $n=$ 3 /group). Scale bar, $25 \mu \mathrm{m}$

\section{Supplementary Files}

This is a list of supplementary files associated with this preprint. Click to download. 
- Tablesupplementary.xls

Page 25/25 